Pacific Journal of Mathematics

HOMOGENIZATION OF REGULAR RINGS OF BOUNDED
INDEX 


\title{
HOMOGENIZATION OF REGULAR RINGS OF BOUNDED INDEX
}

\author{
K. R. Goodearl and D. E. Handelman
}

This paper is about deriving sufficient conditions for a von Neumann regular ring of bounded index of nilpotence to split into a direct product of homogeneous factors, and with deriving necessary and sufficient conditions for such a ring to be biregular. (Most of the results are actually proved in somewhat greater generality, for regular rings whose primitive factor rings are artinian, or for regular rings which are subdirect products of artinian rings.) The splitting of a regular ring $R$ of bounded index into a direct product of homogeneous factors is obtained from purely numerical hypotheses on the set $\mathscr{L}$ of lengths of simple artinian factor rings of $R$. For example, this holds if no element of $\mathscr{C}$ is a positive integral linear combination of other elements of $\mathscr{L}$. In case $R$ is biregular, it suffices to assume that no element of $\mathscr{L}$ is a multiple of any other element of $\because$. Under similar hypotheses on a particular element $t \in \mathscr{Z}$, it is proved that $R \cong R_{1} \times R_{\text {: }}$ such that all simple artinian factor rings of $R_{1}$ have length $t$ (i.e., $R_{1}$ is homogeneous of index $t$ ) and no simple artinian factor rings of $R_{2}$ have length $t$. In the latter part of the paper, topological criteria are derived for $R$ to be biregular. Namely, $R$ is biregular if and only if the prime ideal spectrum of $R$ is Hausdorff, if and only if the set of extreme pseudo-rank functions on $R$ is compact.

All rings in this paper are associative with unit. We use $M_{n}(R)$ to denote the ring of all $n \times n$ matrices over a ring $R$. If $R$ is a simple artinian ring, we use $\ell(R)$ to denote the composition series length of $R$ as a right (or left) $R$-module.

Definition. The index of a nilpotent element $x$ in a ring $R$ is the smallest positive integer $n$ such that $x^{n}=0$. The index of $R$ is the supremum of the indices of all nilpotent elements of $R$. If this supremum is finite, then $R$ is said to have bounded index (or finite index). If $R$ is a regular ring of bounded index $n$, then all indecomposable factor rings of $R$ are simple artinian of length at most $n$, by [5, Theorem 2.3] or [4, Theorem 7.9]. In particular, every regular ring of bounded index is a subdirect product of simple artinian rings. Similar results also hold for any regular ring $R$ whose primitive factor rings are artinian, in which case all indecomposable factor rings of $R$ are simple artinian, by [6, Theorem 3.4] 
or [4, Corollary 6.7].

DEFINITION. An abelian regular (or strongly regular) ring is a regular ring in which all idempotents are central. Equivalently, an abelian regular ring is a resular ring with no nonzero nilpotent elements. Thus a regular ring is abelian if and only if it has index 1 .

DEFinition. Let $R$ be a regular ring of bounded index $n$. We say that $R$ is homogeneous if all primitive factor rings of $R$ also have index $n$. Since all primitive factor rings of $R$ are simple artinian, this is equivalent to requiring that all simple artinian factor rings of $R$ have length $n$. As the following theorem shows, every homogeneous regular ring of index $n$ can be expressed as an $n \times n$ matrix ring over a regular ring of index 1 .

THEOREM 1. (Kaplansky) Let $R$ be a regular ring, and let $n$ be a positive integer. Then $R \cong M_{n}(S)$ for some abelian regular ring $S$ if and only if all primitive factor rings of $R$ are artinian of length $n$.

\section{Proof. [5, Theorem 4.2] or [4, Theorem 7.14].}

It is quite easy to construct regular rings of bounded index which cannot be decomposed into direct products of homogeneous rings, as follows. Start with a numerical relation of the form $m_{1} t_{1}+\cdots+$ $m_{k} t_{k}=t$, where $m_{1}, \cdots, m_{k}, t_{1}, \cdots, t_{k}, t$ are positive integers. Choose a field $F$, and set $T=M_{t}(F)$. In view of the relation $m_{1} t_{1}+\cdots+$ $m_{k} t_{k}=t$, we see that $T$ has a block diagonal subring $S \cong S_{1} \times \cdots \times S_{k}$, where $S_{i}=M_{t_{i}}(F)$. Now let $R$ be the ring consisting of all those sequences $\left\{x_{n}\right\}$ of elements of $T$ for which there exists an index $j$ such that $x_{j} \in S$ and $x_{j}=x_{j+1}=x_{j+2}=\cdots$. It is clear that $R$ is a regular ring of index $t$. Defining $e_{1}, e_{2}, \cdots \in R$ so that $e_{i i}=1$ and $e_{i n}=0$ for all $n \neq i$, we see that these $e_{i}$ are orthogonal central idempotents in $R$, that $\oplus e_{i} R$ is a two-sided ideal of $R$, and that each $e_{i} R \cong T$. However, $R /\left(\oplus e_{i} R\right) \cong S \cong S_{1} \times \cdots \times S_{k}$, hence $R$ has exactly one simple artinian factor ring of length $t_{i}$ for each $i=1, \cdots, k$. Consequently, we conclude that $R$ cannot be isomorphic to a direct product of homogeneous rings.

To summarize this example, given any relation $m_{1} t_{1}+\cdots+$ $m_{k} t_{k}=t$ among positive integers $m_{1}, \cdots, m_{k}, t_{1}, \cdots, t_{k}, t$, there exists a regular ring $R$ of index $t$ such that the set of lengths of simple artinian factor rings of $R$ is $\left\{t, t_{1}, \cdots, t_{k}\right\}$ and such that $R$ is not isomorphic to a direct product of homogeneous regular rings of bounded index. We prove in Corollary 7 that if no (nontrivial) such 
numerical relations hold among the lengths of the simple artinian factor rings of a regular ring $R$ of bounded index, then $R$ is isomorphic to a direct product of homogeneous rings. Our methods are based on analyzing the pseudo-rank functions which arise on artinian factor rings of $R$, as follows.

Definition. A pseudo-rank function on a regular ring $R$ is a map $N: R \rightarrow[0,1]$ such that

(a) $N(1)=1$.

(b) $N(x y) \leqq N(x), N(y)$ for all $x, y \in R$.

(c) $N(e+f)=N(e)+N(f)$ for all orthogonal idempotents $e, f \in R$. A rank function on $R$ is a pseudo-rank function $N$ such that for all $x \in R$,

(d) $N(x)=0$ if and only if $x=0$.

The kernel of a pseudo-rank function $N$ is the set $\operatorname{ker}(N)=$ $\{x \in R \mid N(x)=0\}$, which is a proper two-sided ideal of $R$, by [2, Lemma 5] or [4, Proposition 16.7]. The kernel of a family of pseudo-rank functions on $R$ is defined analogously.

Definition. Given a regular ring $R$, we use $\boldsymbol{P}(R)$ to denote the set of all pseudo-rank functions on $R$. We view $\boldsymbol{P}(R)$ as a subset of the real vector space $\boldsymbol{R}^{R}$, which is given the product topology. As shown in [2, pp. 270, 273] or [4, Proposition 16.17], $\boldsymbol{P}(R)$ is a compact convex subset of $\boldsymbol{R}^{R}$. We shall use both of these properties frequently, particularly compactness. As an alternate description of the topology on $\boldsymbol{P}(R)$, note that a net $\left\{N_{i}\right\} \subseteq \boldsymbol{P}(R)$ converges to some $N \in \boldsymbol{P}(R)$ if and only if $N_{i}(x) \rightarrow N(x)$ for all $x \in R$.

LeMMA 2. Let $R$ be a regular ring, let $t$ be a positive integer, and set

$$
V_{t}=\{N \in \boldsymbol{P}(R) \mid R / \operatorname{ker}(N) \text { is simple artinian of length } t\} .
$$

If $P \in \bar{V}_{t}$, then $R / \operatorname{ker}(P) \cong R_{1} \times \cdots \times R_{k}$ for some simple artinian rings $R_{i}$ and $m_{1} \ell\left(R_{1}\right)+\cdots+m_{k} \ell\left(R_{k}\right)=t$ for some positive integers $m_{i}$.

\section{Proof. Set}

$$
X_{t}=\{N \in P(R) \mid N(x) \in\{0,1 / t, 2 / t, \cdots, 1\} \text { for all } x \in R\},
$$

and note that $X_{t}$ is a closed subset of $P(R)$ which contains $V_{t}$. Thus $P \in X_{t}$, which we claim implies that the ring $\bar{R}=R / \operatorname{ker}(P)$ is artinian. If $\bar{R}$ is not artinian, then it must contain an infinite sequence $g_{1}, g_{2}, \ldots$ of nonzero orthogonal idempotents. By [4, Proposition 2.18], these $g_{i}$ lift to orthogonal idempotents $h_{1}, h_{2}, \cdots$ in $R$. Since each $g_{i} \neq 0$, 
we have $h_{i} \notin \operatorname{ker}(P)$, whence $P\left(h_{i}\right) \geqq 1 / t$. But then

$$
P\left(h_{1}+\cdots+h_{t+1}\right)=P\left(h_{1}\right)+\cdots+P\left(h_{t+1}\right) \geqq(t+1) / t>1,
$$

which is impossible.

Therefore $\bar{R}$ is artinian, as claimed. Then $\bar{R} \cong R_{1} \times \cdots \times R_{k}$ for some simple artinian rings $R_{i}$ of length $t_{i}$.

There exist nonzero orthogonal idempotents $e_{i j} \in \bar{R}$ (for $i=1, \cdots, k$; $\left.j=1, \cdots, t_{i}\right)$ such that $\sum e_{i j}=1$ and $e_{i j} \bar{R} \cong e_{i n} \bar{R}$ for all $i, j, n$. By [4, Proposition 2.18], these idempotents lift to orthogonal idempotents $f_{i j} \in R$ such that $\sum f_{i j}=1$ and each $\bar{f}_{i j}=e_{i j}$. Note that each $P\left(f_{i j}\right)>0$ and that $P\left(f_{i j}\right)=P\left(f_{i n}\right)$ for all $i, j, n$. Since $P \in X_{t}$, we thus find that there exist positive integers $m_{1}, \cdots, m_{k}$ such that $P\left(f_{i j}\right)=m_{i} / t$ for all $i, j$. Consequently,

$$
\sum_{i=1}^{k} m_{i} t_{i} / t=\sum_{i=1}^{k} \sum_{j=1}^{t_{i}} m_{i} / t=\sum_{i=1}^{k} \sum_{j=1}^{t_{i}} P\left(f_{i j}\right)=1,
$$

whence $m_{1} \ell\left(R_{1}\right)+\cdots+m_{k} \ell\left(R_{k}\right)=t$.

LeMma 3. Let $R$ be a regular ring, let $t$ be a positive integer, and set

$$
V_{t}=\{N \in \boldsymbol{P}(R) \mid R / \operatorname{ker}(N) \text { is simple artinian of length } t\} .
$$

Let $P \in P(R)$, and assume that $\operatorname{ker}(P)$ is a maximal two-sided ideal of $R . \quad$ If $\operatorname{ker}\left(V_{t}\right) \leqq \operatorname{ker}(P)$, then $R / \operatorname{ker}(P)$ is simple artinian, and $R$ has simple artinian factor rings $R_{2}, \cdots, R_{k}$ such that $m_{1} \ell(R / \operatorname{ker}(P))+m_{2} \ell\left(R_{2}\right)+\cdots+m_{k} \ell\left(R_{k}\right)=t$ for some positive integers $m_{i}$.

Proof. Set $X_{t}=\{N \in P(R) \mid N(x) \in\{0,1 / t, 2 / t, \cdots, 1\}$ for all $x \in R\}$, and note that $\bar{V}_{t} \subseteq X_{t}$.

We claim that $\operatorname{ker}(N) \leqq \operatorname{ker}(P)$ for some $N \in \bar{V}_{t}$. Suppose not. For each $e \in \operatorname{ker}(P)$, set $W(e)=\{N \in \boldsymbol{P}(R) \mid N(e)>(t-1) / t\}$. Given any $N \in \bar{V}_{t}$, we have $\operatorname{ker}(N) \nsubseteq \operatorname{ker}(P)$ and so $\operatorname{ker}(N)+\operatorname{ker}(P)=R$, by maximality of $\operatorname{ker}(P)$. As a result, there must exist an idempotent $e \in \operatorname{ker}(P)$ such that $1-e \in \operatorname{ker}(N)$, whence $N(e)=1$ and so $N \in W(e)$. Thus $\bar{V}_{t} \subseteq \mathrm{U}_{e \in \operatorname{ker}(P)} W(e)$. Since $\bar{V}_{t}$ is compact and each $W(e)$ is open, there must exist $e_{1}, \cdots, e_{n} \in \operatorname{ker}(P)$ such that $\bar{V}_{t} \subseteq W\left(e_{1}\right) \cup \cdots \cup W\left(e_{n}\right)$. Choose an idempotent $e \in R$ such that $e R=e_{1} R+\cdots+e_{n} R$, and note that $e \in \operatorname{ker}(P)$. Given any $N \in \bar{V}_{t}$, we have $N \in W\left(e_{i}\right)$ for some $i$, whence $N(e) \geqq N\left(e_{i}\right)>(t-1) / t$ and so $N(e)=1$ (because $N \in X_{t}$ ). In particular, $N(1-e)=0$ for all $N \in V_{t}$, hence $1-e \in \operatorname{ker}\left(V_{t}\right)$. On the other hand, $P(1-e)=1$, which contradicts the assumption that $\operatorname{ker}\left(V_{t}\right) \leqq \operatorname{ker}(P)$. 
Therefore $\operatorname{ker}(N) \leqq \operatorname{ker}(P)$ for some $N \in \bar{V}_{t}$, as claimed. According to Lemma $2, R / \operatorname{ker}(N) \cong R_{1} \times \cdots \times R_{k}$ for some simple artinian rings $R_{i}$ and $m_{1} \ell\left(R_{1}\right)+\cdots+m_{k} \ell\left(R_{k}\right)=t$ for some positive integers $m_{i}$. Since $R / \operatorname{ker}(P)$ is simple, we must have $R / \operatorname{ker}(P) \cong R_{j}$ for some $j$, and we may renumber the $R_{i}$ so that $R / \operatorname{ker}(P) \cong R_{1}$.

Definition. We use $\operatorname{Spec}(R)$ to denote the prime ideal spectrum of a ring $R$, that is, the set of all prime ideals of $R$, equipped with the usual hull-kernel topology.

Lemma 4. Let $R$ be a regular ring, let $t$ be a positive integer, and let $J_{t}$ be the intersection of all those two-sided ideals $M$ of $R$ for which $R / M$ is simple artinian of length $t$. If $K \in \operatorname{Spec}(R)$ and $J_{t} \leqq K$, then $R / K$ is simple artinian and $R$ has simple artinian factor rings $R_{2}, \cdots, R_{k}$ such that $m_{1} \ell(R / K)+m_{2}<\left(R_{2}\right)+\cdots+$ $m_{k} \ell\left(R_{k}\right)=t$ for some positive integers $m_{i}$.

Proof. Set $V_{t}=\{N \in \boldsymbol{P}(R) \mid R / \operatorname{ker}(N)$ is simple artinian of length $t$, and note that $J_{t} \leqq \operatorname{ker}\left(V_{t}\right)$. If $M$ is a two-sided ideal of $R$ such that $R / M$ is simple artinian of length $t$, then there exists $N \in V_{t}$ such that $\operatorname{ker}(N)=M$, whence $\operatorname{ker}\left(V_{t}\right) \leqq M$. Thus $\operatorname{ker}\left(V_{t}\right)=J_{t}$.

Inasmuch as $R / J_{t}$ is a subdirect product of simple artinian rings of length $t$, we see that $R / J_{t}$ has bounded index, whence $R / K$ is simple artinian. Then there is some $P \in P(R)$ for which $\operatorname{ker}(P)=K$, and $\operatorname{ker}\left(V_{t}\right) \leqq \operatorname{ker}(P)$, hence we obtain the desired conclusion from Lemma 3.

THEOREM 5. Let $R$ be a regular ring, set $\mathscr{L}=\left\{\measuredangle\left(R^{\prime}\right) \mid R^{\prime}\right.$ is a simple artinian factor ring of $\left.R\right\}$, and let $s \in \mathscr{R}$. Assume the following condition:

(*) If $m_{1} t_{1}+\cdots+m_{k} t_{k}=s$ for some $t_{1}, \cdots, t_{k} \in \mathscr{L}$ and some positive integers $m_{1}, \cdots, m_{k}$, then $k=1$ and $t_{1}=s$.

If $J_{s}$ is the intersection of all those two-sided ideals $M$ of $R$ for which $R / M$ is simple artinian of length $s$, then $R / J_{s} \cong M_{s}(S)$ for some abelian regular ring $S$.

Proof. Applying (*) to Lemma 4, we see that if $K \in \operatorname{Spec}(R)$ and $J_{s} \leqq K$, then $R / K$ is simple artinian of length $s$. Thus all primitive factor rings of $R / J_{s}$ are simple artinian of length $s$, whence Theorem 1 says that $R / J_{s} \cong M_{s}(s)$ for some abelian regular ring $S$.

TheOREM 6. Let $R$ be a regular ring which is a subdirect product of artinian rings, set 


$$
\mathscr{P}=\left\{\ell\left(R^{\prime}\right) \mid R^{\prime} \text { is a simple artinian factor ring of } R\right\},
$$

and let $s \in \mathscr{L}$. Assume the following condition:

(†) Suppose that $m_{1} t_{1}+\cdots+m_{k} t_{k}=t$ for some $t, t_{1}, \cdots, t_{k} \in \mathscr{L}$ and some positive integers $m_{1}, \cdots, m_{k}$. Then $t=s$ if and only if some $t_{i}=s$.

Then $R \cong M_{s}(S) \times T$ for some abelian regular ring $S$ and some regular ring $T$ which has no simple artinian factor rings of length $t$.

Proof. If $\mathscr{L}$ is infinite, then there must exist distinct $t, u \in \mathscr{L}$ which are congruent modulo $s$. If $t<u$, then $m s+t=u$ for some positive integer $m$, which contradicts $(\dagger)$. Thus $\mathscr{L}$ must be finite. Inasmuch as $R$ is a subdirect product of artinian rings, it follows that $R$ has bounded index.

Set $J_{t}=\cap\{M \in \operatorname{Spec}(R) \mid \ell(R / M)=t\}$ for all $t \in \mathscr{L}$, and set $J=\bigcap_{t \neq s} J_{t}$. Note that $J_{s} \cap J=\cap \operatorname{Spec}(R)=0$.

If $K \in \operatorname{Spec}(R)$ and $J_{s} \leqq K$, then by Lemma $4, m_{1} \ell(R / K)+$ $m_{2} t_{2}+\cdots+m_{k} t_{k}=s$ for some $t_{i} \in \mathscr{L}$ and some positive integers $m_{i}$. By $(\dot{\dagger})$, some $t_{i}=s$, which is only possible when $k=1$ and $\ell(R / K)=s$.

Now consider any $K \in \operatorname{Spec}(R)$ such that $J \leqq K$. Since $\mathscr{L}$ is finite, $\Pi_{t \neq s} J_{t} \leqq J \leqq K$ and so $J_{t} \leqq K$ for some $t \neq s$. By Lemma 4, $m_{1} \ell(R / K)+m_{2} t_{2}+\cdots+m_{k} t_{k}=t$ for some $t_{i} \in \mathscr{L}$ and some positive integers $m_{i}$, whence $(\dagger)$ says that $\ell(R / K) \neq s$.

Consequently, no prime ideal of $R$ contains both $J_{s}$ and $J$. Then $J_{s}+J=R$, hence $R \cong\left(R / J_{s}\right) \times(R / J)$. The previous paragraph shows that $R / J$ has no simple artinian factor rings of length $s$. In addition, we have seen that all prime factor rings of $R / J_{s}$ have length $s$, whence Theorem 1 says that $R / J_{s} \cong M_{s}(S)$ for some abelian regular ring $S$.

Corollary 7. Let $R$ be a regular ring which is a subdirect product of artinian rings, and set

$$
\mathscr{C}=\left\{\ell\left(R^{\prime}\right) \mid R^{\prime} \text { is a simple artinian factor ring of } R\right\} \text {. }
$$

Assume the following condition:

(\#) If $m_{1} t_{1}+\cdots+m_{k} t_{k}=t$ for some $t, t_{1}, \cdots, t_{k} \in \mathscr{L}^{P}$ and some positive integers $m_{1}, \cdots, m_{k}$, then $k=1$ and $t_{1}=t$.

Then $\mathscr{P}$ is finite and $R \cong \Pi_{t \in}, M_{t}\left(S_{t}\right)$ for some abelian regular rings $S_{t}$.

The assumption (\#) in Corollary 7 puts boundedness restrictions on $\ell$ (in addition to finiteness). For instance, if $\mathscr{P}$ contains a 
pair of relatively prime integers $t_{1}$ and $t_{2}$, then it is a consequence of (\#) that $t \leqq t_{1} t_{2}-t_{1}-t_{2}$ for all $t \in \mathscr{L}$.

We indicate some cases in which the above results apply. Let $R$ be a regular ring of bounded index, and set

$$
\mathscr{L}=\left\{\ell\left(R^{\prime}\right) \mid R^{\prime} \text { is a simple artinian factor ring of } R\right\} \text {. }
$$

First consider the case when $\mathscr{L}=\{2,3,4\}$. If $J_{2}$ is the intersection of all $M \in \operatorname{Spec}(R)$ for which $\ell(R / M)=2$, then Theorem 5 says that $R / J_{2} \cong M_{2}(S)$ for some abelian regular ring $S$. However, $J_{2}$ need not be a direct summand of $R$, as shown by the examples above. Also, Theorem 6 says that $R \cong M_{3}\left(S^{\prime}\right) \times T$ for some abelian regular ring $S^{\prime}$ and some regular ring $T$ which has no simple artinian factor rings of length 3 . However, $R$ need not be isomorphic to a direct product of homogeneous rings, as shown by the examples above. Now consider the case when $\mathscr{L}=\{3,5,7\}$. In this case, Corollary 7 says that $R \cong M_{3}\left(S_{3}\right) \times M_{5}\left(S_{5}\right) \times M_{7}\left(S_{7}\right)$ for some abelian regular rings $S_{3}, S_{5}, S_{7}$.

Definition. For any ring $R$, we use $B(R)$ to denote the Boolean algebra of all central idempotents in $R$. We use $B S(R)$ to denote the Boolean spectrum of $R$, that is, the spectrum (maximal ideal space) of $B(R)$. The ring $R$ is biregular provided that for any $x \in R$ there is some $e \in B(R)$ such that $R x R=e R$.

If $R$ is a regular rings which is isomorphic to a direct product of homogeneous rings of bounded index, then it follows from Theorem 1 that $R$ must be biregular. Thus we would expect improved versions of Theorems 5 and 6 and Corollary 7 in case the rings involved are biregular. This is indeed so: for the biregular cases of these results, we need only assume that there are no divisibility relations of certain kinds among the lengths of simple artinian factor rings. The proofs in the biregular case are easier as well, since here standard Pierce sheaf methods may be used. We begin with the following easy and well-known lemma, which does not seem to have a suitable reference in the literature.

LEMma 8. Let $R$ be a biregular ring, and let $K$ be a proper two-sided ideal of $R$. Then the following conditions are equivalent:

(a) $K$ is a maximal two-sided ideal of $R$.

(b) $R / K$ is indecomposable (as a ring).

(c) $K=K^{\prime} R$ for some $K^{\prime} \in B S(R)$.

(d) $K \cap B(R) \in B S(R)$ and $K=(K \cap B(R)) R$.

Proof. $\quad(\mathrm{d}) \Rightarrow$ (c) a priori. 
$(c) \Rightarrow(b)$ : Given any central idempotent $e \in R / K$, the biregularity of $R$ impries that there is a central idempotent $f \in B(R)$ such that $\bar{f}=e$. Since $K^{\prime} \in B S(R)$, either $f \in K^{\prime}$ or $1-f \in K^{\prime}$, whence either $e=0$ or $e=1$.

(b) $\Rightarrow$ (a) is immediate from the biregularity of $R$.

$(a) \Rightarrow(d)$ : It is clear from the maximality of $K$ that $K^{\prime}=$ $K \cap B(R)$ belongs to $B S(R)$. Having proved that $(\mathrm{c}) \Rightarrow(\mathrm{b}) \Longrightarrow(\mathrm{a})$, we know that $K^{\prime} R$ is a maximal two-sided ideal of $R$, whence $K^{\prime} R=K$.

LemMa 9 . Let $R$ be a regular ring which is also biregular, let $W$ be a set of positive integers, and let $J_{W}$ be the intersection of all those two-sided ideals $M$ of $R$ for which $R / M$ is simple artinian and $\ell(R / M) \in W$. If $R^{\prime}$ is any simple artinian factor ring of $R / J_{W}$, then $\ell\left(R^{\prime}\right) \mid t$ for some $t \in W$.

Proof. There is a two-sided ideal $K$ of $R$ such that $J_{W} \leqq K$ and $R / K=R^{\prime}$. If $s=\ell\left(R^{\prime}\right)$, then there exist $s \times s$ matrix units $e_{i j} \in R / K$ such that $e_{11}+\cdots+e_{s s}=1$. Choose elements $f_{i j} \in R$ such that $\bar{f}_{i j}=e_{i j}$ for all $i, j$. Inasmuch as $K=(K \cap B(R)) R$ by Lemma 8, there is some central idempotent $g$ of $R$ such that $g \in K$, the $(1-g) f_{i j}$ are $s \times s$ matrix units in $R$, and $(1-g) f_{11}+\cdots+$ $(1-g) f_{s s}=1-g$. Thus $(1-g) R$ is an $s \times s$ matrix ring. Since $g \in K$, we have $1-g \notin K$ and so $1-g \notin J_{W}$. Consequently, there is some two-sided ideal $M$ of $R$ for which $R / M$ is simple artinian, $\ell(R / M)=t \in W$, and $1-g \notin M$. Then $g \in M$, whence $R / M$ is an $s \times s$ matrix ring, and so $s \mid t$.

THEOREM 10. Let $R$ be a regular ring which is also biregular, set

$$
\mathscr{L}=\left\{\measuredangle\left(R^{\prime}\right) \mid R^{\prime} \text { is a simple artinian factor ring of } R\right\},
$$

and let $s \in \mathscr{L}$. Assume that $t \nmid s$ for all $t \in \mathscr{L}$ such that $t \neq s$. If $J_{s}$ is the intersection of all those two-sided ideals $M$ of $R$ for which $R / M$ is simple artinian of length $s$, then $R / J_{s} \cong M_{s}(S)$ for some abelian regular ring $S$.

Proof. Inasmuch as $R / J_{s}$ is a subdirect product of simple artinian rings of length $s$, we see that $R / J_{s}$ has bounded index. Thus if $R^{\prime}$ is any primitive factor ring of $R / J_{s}$, then $R^{\prime}$ is simple artinian. According to Lemma $9, \measuredangle\left(R^{\prime}\right) \mid s$, which by our hypotheses implies that $\ell\left(R^{\prime}\right)=s$. Now apply Theorem 1 .

THEOREM 11. Let $R$ be a regular ring which is also biregular, 
and assume that $R$ is a subdirect product of artinian rings. Set $\mathscr{P}=\left\{\ell\left(R^{\prime}\right) \mid R^{\prime}\right.$ is a simple artinian factor ring of $\left.R\right\}$,

let $s \in \mathscr{C}$, and assume that $s \nmid t$ and $t \nmid s$ for all $t \in \mathscr{L}$ such that $t \neq s$. Then $R \cong M_{s}(S) \times T$ for some abelian regular ring $S$ and some regular ring $T$ which has no simple artinian factor rings of length $s$.

Proof. Let $J_{s}$ be the intersection of all those two-sided ideals $M$ of $R$ for which $R / M$ is simple artinian of length $s$, and let $J$ be the intersection of all those two-sided ideals $M$ of $R$ for which $R / M$ is simple artinian and $\ell(R / M) \neq s$. Note that $R / J_{s}$ has bounded index. Since $R$ is a subdirect product of artinian rings, we see that $J_{s} \cap J=0$.

If $J_{s}+J \neq R$, then $R$ has a maximal two-sided ideal $M$ such that $J_{s}+J \leqq M$. Since $R / J_{s}$ has bounded index, $R / M$ is simple artinian. Then Lemma 9 says that $\ell(R / M) \mid s$ and $\ell(R / M) \mid t$ for some $t \in \mathscr{L}$ such that $t \neq s$. Under our hypotheses, this is impossible.

Therefore $J_{s}+J=R$, whence $R \cong\left(R / J_{s}\right) \times(R / J)$. It is clear that $R / J$ has no simple artinian factor rings of length $s$. Also, Theorem 10 says that $R / J_{s} \cong M_{s}(S)$ for some abelian regular ring $S$.

We would like to thank Walter Burgess for helping to simplify our original proofs of this theorem and the following corollary.

COROLlaRY 12. Let $R$ be a regular ring whose primitive factor rings are artinian, and assume that $R$ is also biregular. Set

$$
\mathscr{L}=\left\{\measuredangle\left(R^{\prime}\right) \mid R^{\prime} \text { is a simple artinian factor ring of } R\right\},
$$

and assume that $s \nmid t$ for all distinct $s, t \in \mathscr{L}$. Then $\mathscr{L}$ is finite and, $R \cong \Pi_{t \in \mathscr{S}} M_{t}\left(S_{t}\right)$ for some abelian regular rings $S_{t}$.

Proof. For each $t \in \mathscr{L}$, Theorem 11 says that there exists a central idempotent $e_{t} \in B(R)$ such that $e_{t} R \cong M_{t}\left(S_{t}\right)$ for some abelian regular ring $S_{t}$ and $\left(1-e_{t}\right) R$ has no simple artinian factor rings of length $t$. Since all simple factor rings of $e_{t} R$ have length $t$, we see that the $e_{t}$ are pairwise orthogonal. If $M$ is any maximal two-sided ideal of $R$, then $R / M$ is artinian and $\ell(R / M)=s$ for some $s \in \mathscr{L}$, whence $1-e_{s} \in M$ and so $e_{s} \notin M$. Thus $\sum e_{t} R$ is not contained in any maximal two-sided ideal of $R$, hence $\sum e_{t} R=R$. Therefore $\mathscr{P}$ is finite and $R \cong \Pi e_{t} R$.

We indicate some cases in which these results apply. Let $R$ be a regular and biregular ring of bounded index, and set 


$$
\mathscr{L}=\left\{\ell\left(R^{\prime}\right) \mid R^{\prime} \text { is a simple artinian factor ring of } R\right\} \text {. }
$$

First consider the case when $\mathscr{L}=\{2,3,5,10\}$. If $J_{5}$ is the intersection of all $M \in \operatorname{Spec}(R)$ for which $\ell(R / M)=5$, then Theorem 10 says that $R / J_{5} \cong M_{5}(S)$ for some abelian regular ring $S$. (This may fail if $R$ is not biregular, because $2+3=5$.) However, $J_{5}$ need not be a direct summand of $R$, as may be shown by an example of the type constructed above. Next consider the case when $\mathscr{L}=\{2,3,8\}$. In this case, Theorem 11 says that $R \cong M_{3}(S) \times T$ for some abelian regular ring $S$ and some regular ring $T$ which has no simple artinian factor rings of length 3 . (This may fail if $R$ is not biregular, because $2+2 \cdot 3=8$.) However, $R$ need not be isomorphic to a direct product of homogeneous rings, as may be shown by an example of the type constructed above. Finally, consider the case when $\mathscr{L}=\{2,3,5\}$. In this case, Corollary 12 says that $R \cong M_{2}\left(S_{2}\right) \times M_{3}\left(S_{3}\right) \times M_{5}\left(S_{5}\right)$ for some abelian regular rings $S_{2}, S_{3}, S_{5}$. (This may fail if $R$ is not biregular, because $2+3=5$.)

Since our results are valid with weaker hypotheses in the biregular case than in the general case, it is of interest to characterize the biregular rings among those regular rings to which our results apply. This we do for regular rings $R$ whose primitive factor rings are artinian. We may view such an $R$ as constructed from a family of simple artinian rings, and the manner in which these simple artinian rings are joined together to form $R$ is indicated by the topology on Spec $(R)$. This topology determines whether or not $R$ is biregular, for, as we prove in Theorem $15, R$ is biregular if and only if $\operatorname{Spec}(R)$ is Hausdorff. It is convenient to proceed by relating biregularity to the following conditions.

Definition. Given modules $A$ and $B$, we use the notation $A \lesssim B$ to mean that $A$ is isomorphic to a submodule of $B$. A regular ring $R$ is said to satisfy the comparability axiom if for any $x, y \in R$, either $x R \lesssim y R$ or $y R \lesssim x R$. A regular ring $R$ is said to satisfy general comparability if for any $x, y \in R$ there is some central idempotent $e \in B(R)$ such that $e x R \lesssim e y R$ and $(1-e) y R \lesssim(1-e) x R$.

Proposition 13. Let $R$ be a regular ring such that all simple homomorphic images of $R$ satisfy the comparability axiom. Then $R$ is biregular if and only if $R$ satisfies general comparability and all prime ideals of $R$ are maximal.

Proof. If $R$ satisfies general comparability and all prime ideals of $R$ are maximal, then $R$ is biregular by [4, Corollary 8.24]. Con- 
versely, assume that $R$ is biregular. By Lemma 8, all prime ideals of $R$ are maximal.

Given elements $x, y \in R$, choose idempotents $g, h \in R$ such that $g R=x R$ and $h R=y R$, and set

$$
\begin{aligned}
& G=\{M \in B S(R) \mid \bar{g}(R / M R) \lesssim \bar{h}(R / M R)\} ; \\
& H=\{M \in B S(R) \mid \bar{h}(R / M R) \lesssim \bar{g}(R / M R)\} .
\end{aligned}
$$

We claim that $G$ and $H$ are open subsets of $B S(R)$. Given $M \in G$, there exist elements $a \in \bar{g}(R / M R) \bar{h}$ and $b \in \bar{h}(R / M R) \bar{g}$ such that $a b=\bar{g}$. Choose $c \in g R h$ and $d \in h R g$ such that $\bar{c}=a$ and $\bar{d}=b$. Then $c d-g \in M R$, hence there exists $f \in M$ such that $c d-g=f(c d-g)$. Consequently, we see that $V=\{K \in B S(R) \mid f \in K\}$ is a clopen subset of $B S(R)$ such that $M \in V \subseteq G$. Thus $G$ is open, and likewise $H$ is open.

For any $M \in B S(R)$, Lemma 8 says that $M R$ is a maximal twosided ideal of $R$, hence by hypothesis $R / M R$ satisfies the comparability axiom. Thus either $\bar{g}(R / M R) \lesssim \bar{h}(R / M R)$ or $\bar{h}(R / M R) \lesssim$ $\bar{g}(R / M R)$, whence $M \in G \cup H$.

Therefore $G \cup H=B S(R)$, hence $B S(R)-G$ and $B S(R)-H$ are disjoint compact subsets of $B S(R)$. Inasmuch as $B S(R)$ is totally disconnected, there must be a clopen set $W \cong B S(R)$ such that $B S(R)-H \subseteq W$ and $B S(R)-G \subseteq B S(R)-W$, so that $W \subseteq G$ and $B S(R)-W \subseteq H$.

Now $W=\{M \in B S(R) \mid e \notin M\}$ for some $e \in B(R)$. For all $M \in W$, we have $M \in G$ and so $\bar{g}(R / M R) \lesssim \bar{h}(R / M R)$. Applying [7, Proposition 3.4] to the ring $e R$, we conclude that $e g R \lessgtr e h R$, that is, $e x R \lesssim e y R$. Similarly, $(1-e) y R \lesssim(1-e) x R$. Therefore $R$ satisfies general comparability.

For many considerations, the topology on the spectrum of a regular ring $R$ is too coarse to distinguish certain needed information. For instance, even if $R$ has bounded index, the maps $M \mapsto$ $\iota(x R / x M)$ (for various $x \in R$ ) need not be continuous. However, the corresponding maps involving $\boldsymbol{P}(R)$, namely $N \mapsto N(x)$, are continuous. In order to effectively utilize this and similar observations, we need topological relations between $\operatorname{Spec}(R)$ and $\boldsymbol{P}(R)$. Specifically, we develop such relations between $\operatorname{Spec}(R)$ and the following subset of $\boldsymbol{P}(R)$.

Definition. Let $K$ be a convex subset of a real vector space. An extreme point of $K$ is any point $x \in K$ which cannot be expressed as a nontrivial convex combination of two distinct points of $K$. In other words, $x$ is an extreme point of $K$ if and only if the only 
convex combinations $x=\alpha y+(1-\alpha) z$ with $0 \leqq \alpha \leqq 1$ and $y, z \in K$ are those for which $\alpha=0, \alpha=1$, or $y=z$. The extreme boundary of $K$, denoted $\partial_{e} K$, is the set of all extreme points of $K$. In situations where $K$ has a topology, we give $\partial_{e} K$ the relative topology.

Proposition 14. Let $R$ be a regular ring whose primitive factor rings are artinian.

(a) There is a continuous bijection $\theta: \partial_{e} P(R) \rightarrow \operatorname{Spec}(R)$ given by the rule $\theta(N)=\operatorname{ker}(N)$.

(b) $\theta$ is a homeomorphism if and only if $\operatorname{Spec}(R)$ is Hausdorff.

Proof. (a) If $N \in \partial_{e} \boldsymbol{P}(R)$, then $R / \operatorname{ker}(N)$ is indecomposable (as a ring) by [4, Lemma 16.24] and so $R / \operatorname{ker}(N)$ is a simple artinian ring. Thus we obtain a map $\theta: \partial_{e} P(R) \rightarrow \operatorname{Spec}(R)$ given by the rule $\theta(N)=\operatorname{ker}(N)$.

If $M \in \operatorname{Spec}(R)$, then $R / M$ is a simple artinian ring, hence there exists a unique rank function on $R / M$. Thus there exists a unique $N \in \boldsymbol{P}(R)$ for which $\operatorname{ker}(N)=M$, and it follows from the uniqueness of $N$ that $N \in \partial_{e} P(R)$. Therefore $\theta$ is a bijection.

If $X$ is a closed subset of $\operatorname{Spec}(R)$, then $X=\{M \in \operatorname{Spec}(R) \mid Y \subseteq M\}$ for some $Y \subseteq R$. As a result,

$$
\theta^{-1}(X)=\left\{N \in \partial_{e} \boldsymbol{P}(R) \mid N(y)=0 \text { for all } y \in Y\right\},
$$

which is a relatively closed subset of $\partial_{e} P(R)$. Therefore $\theta$ is continuous.

(b) Since $\partial_{e} P(R)$ is Hausdorff, necessity is clear. Conversely, assume that $\operatorname{Spec}(R)$ is Hausdorff. We claim that $\partial_{e} P(R)$ must be closed in $\boldsymbol{P}(R)$.

If not, then there is some $N \in \boldsymbol{P}(R)$ which lies in the closure of $\partial_{e} P(R)$ but not in $\partial_{e} P(R)$. There exists a real number $\alpha$ such that $0<\alpha<1$ and distinct $P_{1}, P_{2} \in P(R)$ such that $N=\alpha P_{1}+(1-\alpha) P_{2}$. Note that $\operatorname{ker}(N) \leqq \operatorname{ker}\left(P_{i}\right)$ for each $i$. As a result, we see that $P_{1}$ and $P_{2}$ induce distinct pseudo-rank functions on $R / \operatorname{ker}(N)$. Thus $R / \operatorname{ker}(N)$ is not a simple artinian ring, hence $\operatorname{ker}(N) \notin \operatorname{Spec}(R)$. Consequently, there exist distinct $M_{1}, M_{2} \in \operatorname{Spec}(R)$ such that $\operatorname{ker}(N) \leqq M_{i}$ for each $i$.

Inasmuch as $\operatorname{Spec}(R)$ is Hausdorff, there exist disjoint open sets $V_{1}$ and $V_{2}$ in $\operatorname{Spec}(R)$ such that each $M_{i} \in V_{i}$. Then each $V_{i}=$ $\left\{M \in \operatorname{Spec}(R) \mid X_{i} \nsubseteq M\right\}$ for some $X_{i} \leqq R$. For each $i$, there is some $x_{i} \in X_{i}$ such that $x_{i} \notin M_{i}$. Note that if $M \in \operatorname{Spec}(R)$ and $x_{i} \notin M$, then $M \in V_{i}$. Thus for any $M \in \operatorname{Spec}(R)$, we must have either $x_{1} \in M$ or $x_{2} \in M$.

Since $x_{i} \notin M_{i}$, we have $x_{i} \notin \operatorname{ker}(N)$. Set $W=\{P \in P(R) \mid$ each $\left.P\left(x_{i}\right)>0\right\}$, and note that $W$ is an open subset of $P(R)$ which contains 
$N$. Inasmuch as $N$ lies in the closure of $\partial_{e} \boldsymbol{P}(R)$, there must be some $Q \in \partial_{e} P(R) \cap W$. But then $\operatorname{ker}(Q) \in \operatorname{Spec}(R)$ and each $x_{i} \notin \operatorname{ker}(Q)$, which is impossible.

Thus $\partial_{e} \boldsymbol{P}(R)$ must be closed in $\boldsymbol{P}(R)$, as claimed, whence $\partial_{e} \boldsymbol{P}(R)$ is compact. Therefore $\theta$ is a continuous bijection of a compact space onto a Hausdorff space, hence $\theta$ is a homeomorphism.

The map $\theta$ in Proposition 14 is a continuous bijection of the Hausdorff space $\partial_{e} \boldsymbol{P}(R)$ onto the compact space Spec $(R)$, hence by purely topological considerations, $\theta$ is a homeomorphism if and only if $\partial_{e} P(R)$ is compact and Spec $(R)$ is Hausdorff. Since Proposition 14 shows that $\theta$ is a homeomorphism provided only that $\operatorname{Spec}(R)$ is Hausdorff, it is natural to ask whether it also suffices to assume only that $\partial_{e} \boldsymbol{P}(R)$ is compact. This is true if $R$ has bounded index, as Proposition 16 shows, but not in general, by Example 18.

Definition. Given a module $A$ and a positive integer $n$, we use $n A$ to denote the direct sum of $n$ copies of $A$.

THEOREM 15. If $R$ is a regular ring whose primitive factor rings are artinian, then the following conditions are equivalent:

(a) $R$ is biregular.

(b) $R$ satisfies general comparability.

(c) $\operatorname{Spec}(R)$ is Hausdorff.

Proof. By [1, Theorem 1] or [4, Theorem 6.10], $R$ is unitregular. Consequently, all factor rings of $R$ are unit-regular.

(a) $\Leftrightarrow($ b) by Proposition 13 .

(b) $\Rightarrow(c)$ : According to [4, Theorem 8.25], $\operatorname{Spec}(R)$ is homeomorphic to $B S(R)$, whence $\operatorname{Spec}(R)$ is Hausdorff.

(c) $\Rightarrow($ a): According to Proposition 14, the rule $\theta(N)=\operatorname{ker}(N)$ defines a homeomorphism $\theta$ of $\partial_{e} \boldsymbol{P}(R)$ onto $\operatorname{Spec}(R)$.

Given $x \in R$, set $X_{n}=\left\{N \in \partial_{e} P(R) \mid N(x) \leqq 1 / n\right\}$ and $K_{n}=\operatorname{ker}\left(X_{n}\right)$ for all $n=1,2, \cdots$. Note that $K_{1} \leqq K_{2} \leqq \cdots$. Clearly $X_{n}$ is closed in $\partial_{e} P(R)$, hence $\theta\left(X_{n}\right)$ is closed in $\operatorname{Spec}(R)$, so that $\theta\left(X_{n}\right)=$ $\left\{M \in \operatorname{Spec}(R) \mid K_{n} \leqq M\right\}$.

We claim that $x \in \cup K_{n}$. If not, then there exists $K \in \operatorname{Spec}(R)$ such that $\cup K_{n} \leqq K$ but $x \notin K$. Choose an integer $k>\ell(R / K)$. Inasmuch as $N(x) \leqq 1 / k$ for all $N \in X_{k}$, we infer that $k(x R / x M) \lesssim R / M$ for all $M \in \theta\left(X_{k}\right)$. Applying [4, Theorem 4.19] to the unit-regular ring $R / K_{k}$, we obtain $k\left(x R / x K_{k}\right) \lesssim R / K_{k}$, and consequently $k(x R / x K) \lesssim$ $R / K$. Since $\ell(R / K)<k$, it follows that $x R / x K=0$, which contradicts the fact that $x \notin K$. Thus $x \in \cup K_{n}$, as claimed.

Therefore $x \in K_{n}$ for some $n$. Set $X=\left\{N \in \partial_{e} P(R) \mid N(x)=0\right\}$, 
and note that $X$ is closed in $\partial_{e} P(R)$. Given any $N \in \partial_{e} P(R)$ for which $N(x)<1 / n$, we have $N \in X_{n}$ and $x \in K_{n}=\operatorname{ker}\left(X_{n}\right)$, whence $N \in X$. Thus we also have $X=\left\{N \in \partial_{e} P(R) \mid N(x)<1 / n\right\}$, so that $X$ is a relatively open subset of $\partial_{e} P(R)$.

Consequently, $\theta(X)$ is a clopen subset of $\operatorname{Spec}(R)$, hence there exists $e \in B(R)$ such that $\theta(X)=\{M \in \operatorname{Spec}(R) \mid e \in M\}$. In addition, we have $\theta(X)=\{M \in \operatorname{Spec}(R) \mid x \in M\}$, from which we conclude that $e R=R x R$.

Therefore $R$ is biregular.

Comparing Theorem 15 with Proposition 14 brings up the following question: If $R$ is a regular ring whose primitive factor rings are artinian, and if $\partial_{e} \boldsymbol{P}(R)$ is compact, then is $R$ biregular? We show that this is true in case $R$ has bounded index, but not in general.

Proposition 16. Let $R$ be a regular ring of bounded index, and let $\theta: \partial_{e} \boldsymbol{P}(R) \rightarrow \operatorname{Spec}(R)$ be the continuous bijection given by the rule $\theta(N)=\operatorname{ker}(N)$. Then $\theta$ maps all compact subsets of $\partial_{e} \boldsymbol{P}(R)$ onto closed subsets of $\operatorname{Spec}(R)$.

Proof. Let $W \leqq \partial_{e} \boldsymbol{P}(R)$ be compact, and note that the closure of $\theta(W)$ is the set $\{M \in \operatorname{Spec}(R) \mid \operatorname{ker}(W) \leqq M\}$. Thus it suffices to show that if $M \in \operatorname{Spec}(R)-\theta(W)$, then $\operatorname{ker}(W) \nsubseteq M$.

Note that $\operatorname{ker}(N) \neq M$ for all $N \in W$, whence $\operatorname{ker}(N) \not \equiv M$ for all $N \in W$. Inasmuch as $R$ has bounded index, the set $\mathscr{L}=$ $\{\ell(R / K) \mid K \in \operatorname{Spec}(R)\}$ is finite, hence we may choose an integer $t>\max (\mathscr{L})$. Then for all $N \in \partial_{e} \boldsymbol{P}(R)$ and $x \in R$, either $N(x)=0$ or $N(x)>1 / t$.

For each $x \in R-M$, set $V(x)=\{N \in \boldsymbol{P}(R) \mid N(x)<1 / t\}$, which is an open subset of $\boldsymbol{P}(R)$. Inasmuch as $\operatorname{ker}(N) \geqq M$ for all $N \in W$, we see that these $V(x)$ 's cover $W$. By compactness, there exist $x_{1}, \cdots, x_{n} \in R-M$ such that $W \subseteq V\left(x_{1}\right) \cup \cdots \cup V\left(x_{n}\right)$. There exist $y_{1}, \cdots, y_{n-1} \in R$ such that the element $z=x_{1} y_{1} x_{2} y_{2} \cdots x_{n-1} y_{n-1} x_{n}$ lies in $R-M$. Given any $N \in W$, we have $N \in V\left(x_{i}\right)$ for some $i$, whence $N(z) \leqq N\left(x_{i}\right)<1 / t$ and so $N(z)=0$. Thus $z \in \operatorname{ker}(W)$, whence $\operatorname{ker}(W) \not M$, as desired.

THeOREM 17. Let $R$ be a regular ring of bounded index. Then $R$ is biregular if and only if $\partial_{e} \boldsymbol{P}(R)$ is compact.

Proof. If $R$ is biregular, then $\operatorname{Spec}(R)$ is Hausdorff. In this case, Proposition 14 shows that $\partial_{e} \boldsymbol{P}(R)$ is homeomorphic to $\operatorname{Spec}(R)$, whence $\partial_{e} \boldsymbol{P}(R)$ is compact. 
Conversely, assume that $\partial_{e} \boldsymbol{P}(R)$ is compact. In view of Propositions 14 and 16, we see that the rule $\theta(N)=\operatorname{ker}(N)$ defines a homeomorphism $\theta$ of $\partial_{e} P(R)$ onto $\operatorname{Spec}(R)$. Thus $\operatorname{Spec}(R)$ must be Hausdorff, whence Theorem 15 says that $R$ is biregular.

EXAMPLE 18. There exists a regular ring $R$, whose primitive factor rings are artinian, such that $\partial_{e} \boldsymbol{P}(R)$ is compact but $R$ is not biregular.

Proof. Choose a field $F$, set $R_{n}=M_{n}(F)$ for all $n=1,2, \cdots$, and set $J=\bigoplus R_{n}$, which is a two-sided ideal of $\Pi R_{n}$. Choose an idempotent $e \in \Pi R_{n}$ such that $\operatorname{rank}\left(e_{n}\right)=1$ for all $n$, and set $R=$ $F e+F(1-e)+J$. It is clear that $R$ is a regular subring of $\Pi R_{n}$. Observing that every central idempotent of $R$ is also central in $\Pi R_{n}$, we see that there does not exist $f \in B(R)$ such that $f R=R e R$, whence $R$ is not biregular.

For each $n$, let $g_{n}$ denote the identity element of $R_{n}$. Then $\left(1-g_{n}\right) R$ is a maximal two-sided ideal of $R$ and $R /\left(1-g_{n}\right) R \cong R_{n}$. Also, $M_{1}=F e+J$ and $M_{2}=F(1-e)+J$ are maximal two-sided ideals of $R$ such that $R / M_{1} \cong R / M_{2} \cong F$. Checking that $M_{1}, M_{2}$, and the $\left(1-g_{n}\right) R$ are the only prime ideals of $R$, we find that all primitive factor rings of $R$ are artinian.

In view of Proposition 14, $\partial_{e} \boldsymbol{P}(R)=\left\{Q_{1}, Q_{2}, P_{1}, P_{2}, P_{3}, \cdots\right\}$, where $\operatorname{ker}\left(Q_{i}\right)=M_{i}$ for each $i=1,2$ and $\operatorname{ker}\left(P_{n}\right)=\left(1-g_{n}\right) R$ for all $n=$ $1,2, \cdots$. We claim that $P_{n} \rightarrow Q_{1}$ in $\boldsymbol{P}(R)$.

Given any $x \in R$, we have $x=\alpha e+\beta(1-e)+y$ for some $\alpha, \beta \in F$ and some $y \in J$. Note that $Q_{1}(x)=\operatorname{rank}(\beta)$ (we are viewing $\beta$ as a $1 \times 1$ matrix). Choose a positive integer $k$ such that $y_{n}=0$ for all $n>k$. Then we infer that $P_{n}(x)=[\operatorname{rank}(\alpha)+(n-1) \operatorname{rank}(\beta)] / n$ for all $n>k$, whence $P_{n}(x) \rightarrow Q_{1}(x)$.

Thus $P_{n}(x) \rightarrow Q_{1}(x)$ for all $x \in R$, hence $P_{n} \rightarrow Q_{1}$ in $P(R)$, as claimed. As a result, we conclude that $Q_{1}$ is the only limit point of the set $\left\{P_{1}, P_{2}, \cdots\right\}$, and consequently that $\partial_{e} P(R)$ is closed in $\boldsymbol{P}(R)$. Therefore $\partial_{e} \boldsymbol{P}(R)$ is compact.

\section{REFERENCES}

1. J. W. Fisher and R. L. Snider, Rings generated by their units, J. Algebra, 42 (1976), $363-368$.

2. K. R. Goodearl, Simple regular rings and rank functions, Math. Annalen, 214 (1975), 267-287.

3. - Completions of regular rings, Math. Annalen, 220 (1976), 229-252.

4. - Von Neumann Regular Rings, London (1979), Pitman.

5. I. Kaplansky, Topological representations of algebras. II, Trans. Amer. Math. Soc., $68(1950), 62-75$. 
6. J. Levitzki, On the structure of algebraic algebras and related rings, Trans. Amer. Math. Soc., 74 (1953), 384-409.

7. R.S. Pierce, Modules over commutative regular rings, Memoirs Amer. Math. Soc., No. 70 (1967).

Received August 21, 1978 and in revised form February 26, 1979.

UNIVERSITY OF UTAH

Salt Lake City, Utah 84112

AND

UNIVERSITY OF OTTAWA

Ottawa, Ontario KIN 9B4 Canada 


\section{PACIFIC JOURNAL OF MATHEMATICS}

\section{EDITORS}

DONALD BABBITT (Managing Editor)

University of California

Los Angeles, California 90024

Hugo RossI

University of Utah

Salt Lake City, UT 84112

C. C. MOORE and ANDrew OGG

University of California

Berkeley, CA 94720
J. DUgundjI

Department of Mathematics University of Southern California Los Angeles, California 90007

R. FinN aNd J. Milgram Stanford University Stanford, California 94305

\section{ASSOCIATE EDITORS}

E. F. BECKENBACH

B. H. NeUMANN

F. WOLF

K. YosHIDA

\section{SUPPORTING INSTITUTIONS}

UNIVERSITY OF BRITISH COLUMBIA CALIFORNIA INSTITUTE OF TECHNOLOGY UNIVERSITY OF CALIFORNIA MONTANA STATE UNIVERSITY UNIVERSITY OF NEVADA, RENO NEW MEXICO STATE UNIVERSITY OREGON STATE UNIVERSITY UNIVERSITY OF OREGON
UNIVERSITY OF SOUTHERN CALIFORNIA STANFORD UNIVERSITY UNIVERSITY OF HAWAII UNIVERSITY OF TOKYO UNIVERSITY OF UTAH WASHINGTON STATE UNIVERSITY UNIVERSITY OF WASHINGTON 


\section{Pacific Journal of Mathematics}

\section{Vol. 84 , No. 1 \\ May, 1979}

Michael James Beeson, Goodman's theorem and beyond ...............

Robert S. Cahn and Michael E. Taylor, Asymptotic behavior of multiplicities

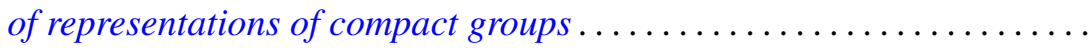

Douglas Michael Campbell and Vikramaditya Singh, Valence properties of

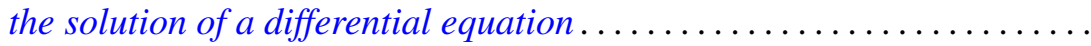

J.-F. Colombeau, Reinhold Meise and Bernard Perrot, A density result in spaces of Silva holomorphic mappings .....................

Marcel Erné, On the relativization of chain topologies .................

Le Baron O. Ferguson, Uniform and $L_{p}$ approximation for generalized

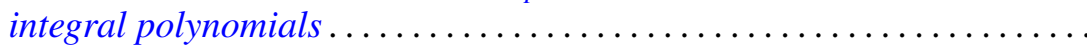

Kenneth R. Goodearl and David E. Handelman, Homogenization of regular rings of bounded index..................................

Friedrich Haslinger, A dual relationship between generalized

Abel-Gončarov bases and certain Pincherle bases .................

Miriam Hausman, Generalization of a theorem of Landau. .

Makoto Hayashi, 2-factorization in finite groups

Robert Marcus, Stochastic diffusion on an unbounded domain ........... 143

Isabel Dotti de Miatello, Extension of actions on Stiefel manifolds....

C. David (Carl) Minda, The hyperbolic metric and coverings of Riemann surfaces...

Somashekhar Amrith Naimpally and Mohan Lal Tikoo, On $T_{1}$-compactifications.

Chia-Ven Pao, Asymptotic stability and nonexistence of global solution for a semilinear parabolic equation ....

Shigeo Segawa, Harmonic majoration of quasibounded type ...

Sze-Kai Tsui and Steve Wright, The splitting of operator algebras ...

Bruce Williams, Hopf invariants, localization and embeddings of Poincaré complexes....

Leslie Wilson, Nonopenness of the set of Thom-Boardman maps ...

Alicia B. Winslow, There are $2^{\mathrm{c}}$ nonhomeomorphic continua in $\beta R^{n}-R^{n}$ 